\title{
Impact of nursing staff and patient education in improving inpatient heart failure care: a closed audit cycle
}

\author{
Authors: Zia Mehmood, Abdullah Abdullah and Andrew Clark
}

\section{Introduction}

The most cost-effective method of delivering heart failure care is by managing patients on cardiology wards with close monitoring of patient's renal functions, weight and fluid balance during diuretic therapy. Better inpatient heart failure care improves patient outcomes. In 2017, a retrospective audit of daily biochemical profile (BCP), weight and fluid balance was undertaken for patients admitted with decompensated heart failure for diuretic therapy to the cardiology department in Castle Hill Hospital.

\section{Methodology}

Data sources included fluid balance charts, weight charts, heart failure charts and our electronic system to check BCP. To assess the quality of fluid balance recorded, a scoring system was designed. The fluid balance chart was scored out of 5 , with a point for each component ie accurate date and patient details, running input, running output, totals added up accurately at the end of each day, and documented fluid balance from last day. A fluid chart score of at least 4 reflected a high standard of documentation. We also looked at the nursing documentation compliance on our separate departmental heart failure chart for these variables.

\section{Results}

\section{Cycle 1 results and discussion}

Between January and March 2017, 606 days of heart failure care was administered. Daily weight was recorded on $79 \%$ of the days, BCP on $70 \%$, and fluid balance on $69 \%$. Only $49 \%$ of fluid charts had a score 4 or above. Documentation compliance on our separate departmental heart failure chart was suboptimal at $59 \%$. The results were presented in weekly departmental teaching sessions with cardiology consultants, registrars, business manager, ward sister and auxiliary nurse in attendance.

\section{Interventions}

Issues such as nurse short staffing and inadequate phlebotomy cover were raised. Interestingly, lack of patient compliance for output monitoring/catheterisation was pointed out by the nursing team to be an issue more often than one would think. The following recommendations were put forward and implemented.

> Patient pamphlets were designed with the importance of fluid restriction, compliance with output monitoring, and bed rest clearly laid out. Images of various sized cups with labelled volumes were included to aid patients to accurately control their daily input to 1.5 litres during intravenous diuretic therapy.

$>$ Small teaching sessions were organised for ward staff and auxiliary nurses to revisit the rationale of monitoring these variables.

> Auxiliary staff were designated at the beginning of each shift to ensure bloods were drawn.

\section{Cycle 2 results}

The re-audit was undertaken in December 2018. Fluid balance monitoring improved from $69 \%$ to $81 \%$, biochemical profile monitoring improved from $70 \%$ to $73 \%$. Percentage of fluid chart score of 4 or above increased from $49 \%$ to $77 \%$ reflecting more than $50 \%$ improvement and high standard of fluid balance recording. The compliance with data recording on separate heart failure charts improved significantly from $59 \%$ to $79 \%$ of the days. Despite a dip, the weight monitoring standard remained reasonably good at $72 \%$.

\section{Conclusion}

Education, constructive feedback and teamwork are cost effective and impactful tools in bettering inpatient heart failure care, a condition best treated with a patient-centred multidisciplinary approach. 